\title{
Dysregulated Fibroblast Growth Factor (FGF) Signaling in Neurological and Psychiatric Disorders \\ ${ }^{*}$ Cortney A. Turner ${ }^{1}$, Emine Eren-Koçak ${ }^{2}$, Edny G. Inuii ${ }^{3}$ Stanley J. Watson ${ }^{1,4}$ \& Huda Akil ${ }^{1,4}$ \\ ${ }^{1}$ Molecular \& Behavioral Neuroscience Institute, University of Michigan, Ann Arbor, Ml \\ ${ }^{2}$ Institute of Neurological Sciences and Psychiatry, Hacettepe University, Ankara, Turkey \\ ${ }^{3}$ Sagient Research, San Diego, CA \\ ${ }^{4}$ Department of Psychiatry, University of Michigan, Ann Arbor, MI
}

${ }^{*}$ Corresponding author

Molec \& Behav Neursoci Inst

205 Zina Pitcher Place

University of Michigan

Ann Arbor, Ml. 48108

Phone: (734) 936-2034

Email: caturner@umich.edu 


\section{Abstract}

The role of the fibroblast growth factor (FGF) system in brain-related disorders has received considerable attention in recent years. To understand the role of this system in neurological and psychiatric disorders, it is important to identify the specific members of the FGF family that are implicated, their location and the various mechanisms they can be modulated. Each disorder appears to impact specific molecular players in unique anatomical locations, and all of these could conceivably become targets for treatment. In the last several years, the issue of how to target this system directly has become an area of increasing interest. To date, the most promising therapeutics are small molecule inhibitors and antibodies that modulate FGF receptor (FGFR) function. Beyond attempting to modify the primary players affected by a given brain disorder, it may prove useful to target molecules, such as membranebound or extracellular proteins that interact with FGF ligands or FGFRs to modulate signaling.

KEYWORDS: Hippocampus, Neurological, Psychiatric, Brain, Development, Therapeutics 


\section{Introduction}

The explosion in brain research has not only enhanced our specific knowledge about the brain from molecule to behavior, but it has also changed our view of the very nature of the organ, one that is more capable of self-remodeling and repair than neuroscientists had previously expected. The early emphasis on classical neurotransmitters may have underestimated the critical role of other classes of molecules that not only guide the building of the brain, but also maintain its viability, plasticity and ability to rewire and repair itself.

Over the last three decades, growth factors have emerged as critical players in the development, remodeling and long-term survival of brain structure and function. But they also appear to perform other, more rapid modulatory functions that remain to be fully understood or explored. While the groundbreaking work on Nerve Growth Factor (NGF) [1] had focused on the development of the peripheral nervous system, the discovery of a brain derived neurotrophic factor in 1982, later termed BDNF, began to shift the emphasis towards the central nervous system [2]. But it was not until the late 1980's and early 1990's that a picture began to emerge more clearly regarding the existence of multiple neuronal functions of growth factors from different families, including the target-derived neurotrophins (NGF, BDNF, Neurotrophin-3 or NT3) and Ciliary Neurotrophic Factor (CNTF). In a thoughtful review of the neurotrophin field, Hans Thoenen (1991) essentially laid out the outlines of future research in the area: "The rapid regulation of neurotrophic molecules by neuronal activity using specific transmitter systems suggests that the function of neurotrophic molecules is much more than merely to regulate the survival of neurons during development and to maintain the function of fully differentiated neurons." He went on to add: "The elucidation of the structure and function of new neurotrophic molecules, along with the understanding of their physiological functions, might also open up attractive possibilities for their therapeutic use in the treatment of traumatic and degenerative diseases of the peripheral and central nervous systems" [3].

The role of the Fibroblast Growth Factor Family (FGF) has an even shorter history in the context of neuroscience. As the name suggests, the role of FGFs in skin is better known, and their functions in wound healing much better studied, with over 1000 publications on this topic beginning with the observation that soluble cues from the dermis aid in wound healing. During healing, the skin secretes growth factors which are bound by the support matrix to regulate activity. Indeed, one of the main soluble cues in skin is FGF2. Exogenous FGF2 is known to increase other growth factors and promote healing peripherally [4]. Moreover, an increase in other growth factors following FGF2 administration has been observed during tissue regeneration [5]. Advances in biomedical engineering are allowing the reproduction of the support matrix to recapitulate the cues that drive repair of the skin. Work on the FGF family in this arena and in the area of cancer research promises to provide many tools that might prove useful in the context of neuropsychiatric and neurological disorders.

Our own interest in the role of FGFs in affective disorders began over 10 years ago based on observations in human post-mortem samples [6]. Since then, numerous strands of evidence both in human studies and animal models have come together to implicate the FGF family in a range of brain disorders. Below, we provide an overview of this growth factor family, summarize the evidence pointing to its implication in the pathophysiology of brain disorders and discuss possible opportunities for targeting it to derive novel treatment strategies.

\section{The FGF System}

\subsection{FGF ligands}

To understand the role of the FGF system in neurological and psychiatric disorders, it is useful to examine the role of this system in development. In mammals, there are 18 different FGF ligands than can be secreted. Several of the secretable ligands drive brain development by imparting positional information and regulating other transcription factors involved in patterning. In addition to the secretable ligands, there are four FGFs termed FGF homologous factors 
(FGF11-FGF14) that remain intracellular. Five members of the FGF family are important for cortical patterning: FGF3, FGF8, FGF15, FGF17 and FGF18 [7]. With the involvement of so many FGFs, there is a natural hierarchical organization of function. FGF8 can control FGF17, while FGF8 and FGF17 together can activate numerous transcription factors involved in intracortical wiring and size, whereas FGF15 functions in opposition to their actions [8]. Defects in these FGFs have been suggested to contribute to some of the cortical abnormalities observed in autism spectrum disorders [9].

FGF8 appears to be the main player in midbrain, hindbrain and forebrain patterning [10]. This factor is also capable of regulating FGF receptors, especially in the hippocampal-derived neuronal cells [11]. FGF17 is also involved in the patterning of the frontal cortex through regulation of transcription factor expression $[12,13]$. While some members of the FGF family are involved in patterning and growth, others are critical in specifying the nature of the signaling. For example, FGF22 and FGF7, play a role in excitatory and inhibitory presynaptic differentiation, respectively [14]. Finally, FGF9 was initially identified as glia activating factor [15].

Another ligand, FGF2 (also termed basic FGF) is involved in neurogenesis during brain development [16]. Remarkably, FGF2 has been recently shown to facilitate the formation of functional dopaminergic neurons from stem cells derived from bone marrow [17]. In addition, FGF2 can induce angiogenesis and is a pro-survival, pro-migration and pro-differentiation factor. With large 3' and 5' regions, it is the target of a complex transcriptional regulation by miRNAs and various transcription factors [18].

Two key regulators of FGF2 are CREB and carboxypeptidase E (CPE). Recently, chronic stress in mice sufficient to produce depression-like behavior was shown to decrease CPE along with decreased expression of FGF2 [19]. Furthermore, CPE knock-out mice exhibit depression-like behavior that was reversed by FGF2 administration. CPE likely upregulates FGF2 through extracellular signal-regulated kinase-Sp1. FGF2 can also be regulated by microRNAs, such as miR-15a and miR-16 [20]. In turn, FGF2 regulates other miRNAs, such as miR-134 [21].

In humans, FGF2 can exist in different molecular weights, $18 \mathrm{kDa}, 21 \mathrm{kDa}, 22.5 \mathrm{kDa}$, $24 \mathrm{kDa}$ and $34 \mathrm{kDa}$, translated from alternate initiation codons. The two highest molecular weight (HMW) isoforms do not exist in rodents. The low molecular weight (LMW) 18kDa isoform is found both in the nucleolus and cytoplasm and can be secreted, whereas the other four HMW isoforms remain in the nucleus [22]. Thus, the different isoforms can have distinct functions in different regions and exhibit different expression patterns throughout early brain development. FGF2 does not have a signal peptide for secretion, and the mechanism of secretory export remains unclear. Recently, it was proposed that the $\mathrm{Na} / \mathrm{K}$-ATPase plays a role in the secretion of FGF2 [23]. One particular subunit, ATP1A1 directly recruits FGF2 to the plasma membrane where it can oligomerize and form a lipid pore. Heparin sulfate proteoglycans then help translocate FGF2 across the membrane.

In addition to paracrine/autocrine FGFs, there are endocrine ligands. FGF21 is found in primary neurons known to facilitate glucose and lipid metabolism by interacting with FGFRs and $\beta$-klotho. FGF21 can be increased by mood stabilizers, such as lithium or valproic acid and by phosphorylated Akt. In turn, FGF21 can activate Akt to protect hippocampal neurons from glutamate toxicity [24]. Similarly, FGF23 is increased in serum following lithium treatment in individuals with major depressive disorder, although the nature of the effect in controls is unknown [25]. Secondary effects of FGF21 and FGF23 have also been observed in the brain [26]. For example, overexpression of FGF23 has been found to induce hypophosphatemia. These animals also exhibited impaired learning and memory and reductions in hippocampal markers secondary to the hypophosphatemia [27]. 
The above thumbnail points to the wide range of functions played by the FGF family in the nervous system, via a number of cellular mechanisms, molecular partners and signaling pathways.

\subsection{FGF Receptors}

FGF signaling is mediated through four membrane-bound receptors (FGFR1-FGFR4) which share $46 \%$ amino acid identity. FGFR1 and FGFR4 are primarily neuronal, whereas FGFR2 and FGFR3 are expressed in oligodendrocytes and astrocytes, respectively [28]. FGFR1 is the only FGF receptor to date that can be increased by nuclear levels of FGF2 [29]. Conversely, FGF2 can also be regulated by nuclear FGFR1 [30]. Inside the nucleus, FGFR1 can co-localize with FGF2 in various cell types to form a complex with CREB-binding protein (CBP) to regulate transcription of various genes [31].

FGFR1 is the more abundant receptor in the brain and while primarily neuronal, it is also located on radial glia cells [32]. FGFR1 is necessary for learning and memory, hippocampal growth and pyramidal neurons, whereas FGFR2 is involved in progenitor cell proliferation and learning and memory [33]. FGFR3 has been implicated in the proper formation of the cortex [34]. Taken together the three receptors are responsible for the size of the cortex by maintaining an adequate supply of radial glia [35]. In relation to normal brain development, mutations in FGFR2 can lead to either Apert Syndrome or Crouzon syndrome marked by subcortical changes in the shape of several brain structures independent of changes in the skull [36].

The FGF receptors have three extracellular immunoglobulin-like domains (Igl, Igll and IgIII), a single transmembrane domain and an intracellular tyrosine kinase domain (for review see [37-39]). FGF ligands bind to IgII and IgIII and the linker region between IgII and IgIII, whereas heparan sulfate binds to the basic canyon region of Igll [40]. The receptors dimerize and autophosphorylate after two FGF ligands and two heparan sulfate proteoglycans bind the receptor. The activated receptor signals through three main pathways: PLCY, MAPK and AKT. Additional diversity in this receptor family derives from alternative splicing. Thus, two receptor isoforms can result from the alternate use of exon 8 or exon 9 , resulting in the IIIb or IIIC isoforms respectively. The expression of these two isoforms for FGFR1-3 affects their signaling, ligand selectivity, binding to other partnering proteins and cell-type specificity [41]. The IIIb isoform is more common in early brain development, and there is some indication that activation of the IIlb isoform later in life could have deleterious consequences [42].

\section{FGF System in Neurological Disorders}

Since the FGF system is involved in the formation of those brain circuits in the hippocampus and cortex that are associated with epileptogenesis, it is no surprise that FGF members can play a role in seizures. Indeed, mutations or deletions in FGF8 and FGF17 have been identified in some epileptogenic disorders, probably due to their roles in brain development [43]. More broadly, the FGF family is highly responsive to seizures and FGF1, FGF2, FGF5, FGF7, FGF8, FGF17 and FGF22 have all been shown to be altered in epileptogenesis [43]. In turn, these FGF ligands likely mediate some of the lasting effects of seizures on the brain. In the hippocampus, their post seizure actions include cell death, astrocytosis, damage to the bloodbrain-barrier (BBB), mossy fiber sprouting, aberrant neurogenesis and alteration of excitatory and inhibitory terminals. FGF5 expression is increased shortly after acute seizures, along with an increase in FGF2 and FGFR1 [44]. However, FGF5 appears to have contrasting roles in epilepsy development, as is common in this family. Specifically, it appears to favor astrocytosis while at the same time protecting the BBB. It is also possible that some members of the FGF family could play a protective effect. In rodents, FGF1 exerts an anticonvulsant effect in the kainate model [45], whereas inhibiting FGF22 and activating FGF7 may help alleviate epileptogenesis [43]. The latter may be due to their disparate roles in presynaptic differentiation of glutamate and GABA neurons [14]. 
Perhaps the best studied FGF member in the context of neurological disorders is FGF2. In general, it reduces brain damage and improves function in animal models [43]. However, in epilepsy research, FGF2 has a multi-faceted role. Acute FGF2 can favor seizures while chronic FGF2 can reduce seizure-induced behavioral deficits and cell death. The upregulation of FGF2 following a seizure is fast and transient in the hippocampus and cortex [46]. However, the effects of the higher molecular weight isoforms may be sustained and function to protect the cells from further injury.

FGF2 may also play a role in autoimmune diseases of the brain, such as multiple sclerosis (MS) and lupus. In MS, FGF2 was higher in patients than in controls and higher in relapse patients than in those in remission $[47,48]$. This is thought to be a protective effect based on postmortem findings in lesions [49]. FGF2 is known to regulate myelination, which is decreased in MS [50]. Moreover, FGFR1 and FGFR2 mutant mice exhibit hypomyelination, suggesting that FGF signaling is necessary to increase oligodendrocytes and form myelin sheaths of normal thickness. Overexpression of FGF2 has also been shown to be beneficial in the experimental autoimmune encephalomyelitis model of MS [51]. In lupus, FGF2 is positively correlated with disease activity [52]. A recent study compared the protein levels of various growth factors in the cerebrospinal fluid (CSF) of lupus patients relative to subjects with disrupted BBB, such as MS. FGF2 was one of six upregulated genes that predicted lupus using a weighted algorithm [53].

To address whether the FGF system is altered around the site of the brain injury, we performed a single vehicle microinjection (artificial extracellular fluid) into the dentate gyrus of rats. As shown in Table 1, FGF2 and FGFR1 gene expressions were increased 24 hours following the infusion compared to uninjected control rats (unpublished data). Interestingly, both FGF2 and FGFR1 expression were no longer different from controls after one week. The expression of FGF9, initially termed glia activating factor, BDNF and trkB are also shown. These findings have interesting implications given the various nuclear functions of FGFR1 discussed in Section 2.2. This finding also suggests that microinjections may be traumatic, and that the FGF system may be promoting recovery from the trauma. While studies routinely use vehicle microinjection as a control, interpretations rarely consider the possibility of an interaction between the microinjection process and the experimental manipulations, including the induction of growth factors.

\section{FGF System in Neurodegenerative Disorders}

Several FGF family members have been implicated in Parkinson's disease. Among these, perhaps the most prominent is FGF20. FGF20 is preferentially expressed in the substantia nigra pars compacta (SNpc) and has been shown to enhance the survival of dopaminergic (DA) neurons [54]. The mechanism involves activation of FGFR1Illc and the MAPK pathway. When DA neurons were differentiated from embryonic stem cells, FGF20 acted in synergy with FGF2 to increase dopamine cell number [55]. In the 6-hydroxydopamine lesion model, infusions of FGF20 into the substantia nigra protected against dopamine neuron loss in both the SNpc and striatum and preserved motor function [56]. Since this study implied a protective role of FGF20, it seemed logical that genetic variation in this gene may predispose individuals to Parkinson's disease. To this end, a single nucleotide polymorphism (SNP) in the 3' UTR of FGF20 has been associated with Parkinson's disease risk [57]. The SNP involved affects binding of miR-433 which increases translation of FGF20. More recent work has described an association between FGF20 and $\alpha$-synuclein in sporadic Parkinson's disease [58]. However, this may occur only in certain populations as a study in Northern Han Chinese did not find any differences in FGF20 SNPs [59].

Since FGF20 can act in conjunction with FGF2 to increase dopaminergic neuron number in primate stem cell models [55], much research has also focused on the role of FGF2 in the nigrostriatal pathway. Intrastriatal expression of LMW FGF2 has also been shown to increase 
dopaminergic neuron recovery following a chemically-induced lesion [60]. This increased survival is thought to be the result of signaling through microglia to clean up amyloid $\beta$ [61]. In the context of genetic models, mice deficient in all FGF2 isoforms show reduced dopamine (DA) neuron number. The opposite is true for transgenic mice that overexpress FGF2 [62]. Interestingly, FGFR1 can control the development of dopaminergic neurons in the SNpc. Mice lacking the tyrosine kinase region of FGFR1 in tyrosine hydroxylase cells exhibit a smaller SNpc and fewer DA neurons [63].

FGF2 has also been shown to be beneficial in animal models of Alzheimer's disease. Overexpression of FGF2 restored spatial learning, long-term potentiation, and neurogenesis [64]. The mice also showed a reduction in amyloid $\beta$ through microglial activation. The proposed mechanism is through FGFR1 signaling increasing OX-2 membrane glycoprotein (also termed CD200) [65]. In a mouse model of Alzheimer's disease based on overexpression of a mutant form of amyloid precursor protein (APP23), FGF2 treatment reduced the expression of beta-site amyloid precursor protein-cleaving enzyme 1 (BACE1), the enzyme responsible for the production of amyloid $\beta$ [66]. This reduction was associated with a decrease in amyloid $\beta$ deposition, tau pathology and spatial memory deficits.

\section{FGF System in Psychiatric Disorders}

Several reports have demonstrated alterations in the FGF system in individuals with major depressive disorder (MDD). The first demonstration by Evans et al. (2004) found decreased expression of FGF1, FGF2, FGFR2 and FGFR3 in cortical areas, and increased expression of FGF9 and FGF12 [6]. Five subsequent reports further validated and described differential expression of FGF family members in post-mortem brain (reviewed in [67]). One study found no differences in the plasma levels of FGF2 [68]. However, a recent report on serum of patients with MDD found lower serum FGF2 levels in these patients compared to controls [69]. FGF2 levels did not appear to respond to treatment, but it should be noted that there were seven different medications in the cohort being studied and not all of them have been shown to increase FGF2 levels. This recent study excluded individuals with a family history of MDD and included medication-free patients. The latter is an important difference between the two studies.

Recently, the circadian system was found to be disrupted in the post-mortem brains of individuals with MDD [70]. Therefore, we decided to assess whether FGF2 and FGFR1 exhibit circadian rhythmicity in the rodent hippocampus. For this study, lights were on at $0700 \mathrm{~h}$ and off at $1800 \mathrm{~h}$. As shown in Figure 1, the peak for FGF2 was at $1600 \mathrm{~h}$, whereas the nadir for FGFR1 was at $2400 \mathrm{~h}$ (unpublished data). This suggests that FGFR1 lags FGF2, with the expression pattern of FGF2 similar to the pattern of corticosterone levels in blood.

Several reports in animal models have demonstrated an antidepressant effect of FGF2. The first of these showed an antidepressant effect of intracerebroventricular FGF2 in rats [71]. Subsequent studies in mice using different models for depression have further described the role of FGF2 in depression. FGF2 was antidepressant and rescued deficits induced by a model of depression-like behavior [72]. Antidepressant effects were also observed following FGF2 infusion into the prefrontal cortex in the chronic unpredictable stress model of depression [73]. In this latter study, FGF2 also prevented the stress-induced decrease in glial proliferation. Most recently, chronic mild stress was used to induce depression-like behavior in a stroke model [74]. These animals exhibited less FGF2 (mRNA and protein) in the frontal lobe compared to controls.

The first evidence that FGF2 has an anxiolytic effect was demonstrated by Perez et al. (2009) [75]. This was associated with an increase in the survival of both neurons and glia in the dentate gyrus. Additional work found that early life administration of FGF2 could also decrease anxiety-like behavior in adulthood [76]. This was associated with an altered developmental trajectory of the hippocampus and alterations in gene transcription. Many of the transcripts were 
previously associated with either anxiety or cell survival. Recently, early life FGF2 has been shown to have epigenetic effects. Epigenetic marks can include DNA methylation, histone posttranslational modifications and non-coding RNAs [77]. FGF2 can influence the methylation status of histones and be influenced by histone methylation. Specifically, the association of trimethylated histone protein $\mathrm{H} 3$ at lysine 9 (H3K9me3) at the FGF2 promoter was lower in animals that naturally have less FGF2 [78]. These same animals also have increased anxietylike behavior. Furthermore, FGF2, which is known to decrease anxiety-like behavior, increased the association of H3K9me3 at the FGF2 promoter. It is possible that this epigenetic effect was mediated by FGF2 translocating to the nucleus, as in other model systems [79].

The FGF system has been implicated in the mechanism of action of antidepressants. It is well known now that tricyclic antidepressants and selective serotonin reuptake inhibitors can increase FGF2 levels in cortical neurons [80]. This coincides with a mobilization of the HMW FGF2 from the nucleus to the cytoplasm. More recently, a monoamine oxidase inhibitor has been shown to increase both the low and high molecular weight isoforms of FGF2 in cortical astrocytes [81]. This body of work has led us to conclude that the FGF system in general, and FGF2 in particular are strongly implicated in the regulation of affect. This is based on both human and animal studies, with FGF2 functioning as an endogenous anxiolytic and antidepressant that is downregulated in the brain of severely depressed humans [67].

So far, alterations in FGF2 and FGFR2 have been implicated in bipolar disorder. In a large-scale case-control study in the Chinese Han population, one SNP in FGFR2 (rs111199993) was found to be associated with bipolar disorder [82]. As for FGF2, serum levels were higher in bipolar patients than in controls [83]. Since all of these subjects were in a manic episode, FGF2 levels also correlated with the duration and severity of the mania. Interestingly, mood stabilizers can alter the FGF system by epigenetic mechanisms. The primary mood stabilizer for bipolar disorder, valproate, has recently been shown to increase FGF1 expression by inhibiting HDAC activity [84]. The HDAC enzyme, which removes acetyl groups from histones, typically has an inhibitory role on gene expression. The increase in FGF1 expression involves altered binding of the regulatory factor $\mathrm{X}$ (RFX) transcriptional complex to the FGF1 promoter. Moreover, lithium chloride, another common treatment for bipolar disorder, also increased FGF1 expression by inhibiting GSK3. Like FGF1, FGF2 also has RFX sites in its promoter. Therefore, mood stabilizers may explain the increase in FGF2 in bipolar patients.

As reviewed by Terwisscha van Scheltinga et al. (2013), the FGF system has also been implicated in schizophrenia [85]. A large association study found that genetic variation in FGFR2 was associated with an increased risk of developing schizophrenia [86]. Subsequently, a genome-wide study strongly implicated a SNP near FGFR1 in schizophrenia [87]. In postmortem brain analyses of gene expression, FGFR1 was found to be increased and FGFR2 was found to be decreased in schizophrenia $[88,89]$. In the periphery, FGF2 levels were increased in schizophrenic patients with high negative symptoms compared to controls [90]. Since a direct interaction has been shown between FGF receptors and adenosine A2 receptors, this suggests that FGF signaling could antagonize dopamine D2 receptors as well [91].

\section{Targeting the FGF System}

Given the range of functions of the FGF system and its implication in a number of neurological and psychiatric disorders, there is growing interest in finding strategies to modify its actions in order to ameliorate these disorders. While profiting from the broad interest in the FGF system outside the brain, these efforts are still in the early phases in terms of CNS applications and present a number of challenges. The receptors, while readily identifiable and targetable are also a point of convergence for the actions of a large family of FGF ligands for the entire organism, and the consequences of their activation or blockade need to be carefully evaluated. The diversity of molecules that partner with the FGF ligands and their receptors offer great targets of opportunity but also require more in-depth analyses of which molecule should be 
targeted for altering particular neural dysfunctions or disease states. Below we describe some of the available tools and a synopsis of what is currently known about their neural actions.

\section{$\underline{6.1 \text { Antibodies }}$}

While specific antibodies targeted against any of the players in the FGF family may prove to be highly useful, a major challenge for brain disorders lies in the fact that they need to penetrate the blood-brain-barrier, at least in sufficient amounts as to be effective. Since 2008, the majority of the FGF ligand antibodies have focused on the endocrine FGFs. Anti-FGF23 may help in the treatment of hypophosphatemia, and anti-FGF19 may help in the treatment of hepatocellular carcinoma [92]. However, the endocrine ligands may not be as central to the neurological and psychiatric disorders as the classical paracrine-acting FGFs that we discussed above.

Much of the work in generating antibodies has derived from cancer research, where blockade of some FGFs or their receptors is sought as a means of reducing or reversing the progress of cancer. Although the development of these antibodies has been for oncology applications, there is the potential to explore their use in the context of brain disorders. While a few of these FGF antibodies have targeted ligands implicated in brain disorders, such as FGF2 most of the antibody patent filings since 2008, have focused on FGF receptors (reviewed in [92]). All of the antibodies in effect block binding of the natural ligands, depending on the isoform that is targeted. However, these antibodies have largely fallen short in Phase I clinical trials due to lack of efficacy in only targeting a single FGF receptor.

A promising alternative approach lies in the realm of FGF-traps. These are soluble portions of FGF receptors fused with the Fc fragments of an antibody that act as decoy receptors. These antibodies then have the ability to sequester specific FGF ligands [92]. One of these traps is the FGFR1-Fc designed by Five Prime Therapeutics, Inc. which is in Phase II trials for endometrial cancer (reviewed in [92]).

\section{$\underline{6.2 \text { Peptides }}$}

Using peptide mimetics may be promising in that it might circumvent the specificity issue by targeting specific molecular partners that might be present only in the nervous system, or might be particularly dysregulated in the context of a specific brain disorders.

Neural cell adhesion molecule, NCAM, is a transmembrane protein that can interact with FGF receptors, independent of FGF ligands, and regulate synaptic plasticity. Originally known for its axonal growth properties, it is fairly ubiquitous in the brain and has several peptide mimetics [93]. The F and G loop (FGL) peptide has been the best studied of these and can activate FGF receptors, albeit with a lower affinity than FGF ligands [94]. Although many of its functions have been previously reviewed [67], the peptide has recently been shown to enhance synaptic transmission. Another peptide, enreptin can interact with both FGF receptors and NCAM [95], and this dual specificity agonist action may result in a greater response than peptides that only interact with the FGF receptor. This peptide crosses the BBB and has been shown to reduce inflammation, enhance memory, and be protective in models of Alzheimer's disease and multiple sclerosis [95].

There are also peptides derived from other proteins that interact with FGF receptors. The membrane glycoprotein CD200 can directly interact with FGFR1. The oxifin1 peptide derived from CD200 mimicked its neuritogenic and survival-promoting effects [96]. Proteins of the cell adhesion molecule (CAM) family, such as nectin-1, can also bind and activate FGF receptors [97]. CAMs signal through FGF receptors by trans-homophilic/heterophilic interactions of CAMs on opposite cells. CAMs interact with each other and then interact in cis with FGF receptors. Nectin-1 is prominently expressed in brain, including the hippocampus. Furthermore, nectide is a peptide derived from nectin- 1 which can mimic its effects, including those on cell survival [97]. 


\section{Conclusions}

In summary, the FGF system is complex, with a range of biological functions ranging from nuclear to secretory proteins. Beyond the breadth and diversity of the family itself, it engages a wide range of molecular partners that modulate its actions in ways that are yet to be fully understood or integrated. It is clear that the orchestration between all the molecular partners and their cross-regulation is greatly affected by context, including the stage of development or aging, the cellular milieu, and the presence of various modulating factors and interacting factors. Moreover, it is evident that members of the FGF family can function in multiple time domains, guiding entire development programs and yet at least in some cases responding instantaneously to guide behavioral responsiveness. Members of this family, such as FGF2 can promote neurogenesis, help in recovery from injury, enhance learning and memory and protect against negative affect, while others modulate functions such as feeding and metabolic activity. The FGF system has been implicated in neurological disorders, such as epilepsy, neurodegenerative disorders, such as Parkinson's disease, and psychiatric disorders, such as major depression. FGF ligands may mediate their effects through epigenetic mechanisms, whereas FGF receptors may have a multitude of effects through interactions with other partnering proteins. The search is on for novel modulators of the system, such as small molecule inhibitors, proteins/peptides, and antibodies. An interesting tension is that the focus of the pharmaceutical industry has been aimed at inhibiting this system in order to control cancer. By contrast, positive regulators are likely needed to ameliorate neurological and psychiatric disorders. Finding the appropriate combination of tools that work in the brain without causing untoward side effects throughout the body is a major challenge that will require a much greater depth of understanding of the FGF family in the context of neural circuits, both under healthy and pathological conditions. 


\section{Acknowledgements}

The authors declare no competing financial interests. This work was funded by the Hope for Depression Research Foundation, NIMH (R01 MH104261) and the Office of Naval Research (N00014-12-1-0366). The authors are members of the Pritzker Neuropsychiatric Disorders Research Consortium, which is supported by the Pritzker Neuropsychiatric Disorders Research Fund L.L.C. A shared intellectual property agreement exists between this philanthropic fund and the University of Michigan, Stanford University, the Weill Medical College of Cornell University, HudsonAlpha Institute of Biotechnology and the University of California at Irvine, to encourage the development of appropriate findings for research and clinical applications. 


\section{Figure Legend}

Figure 1: Gene expression of FGF2 and FGFR1 in the hippocampus exhibit circadian rhythms in opposite directions $†$. Lights were on at $0700 \mathrm{~h}$ and off at $1800 \mathrm{~h}$ (unpublished data). A) FGF2 expression showed peak expression at $1600 \mathrm{~h}$ and trough expression at $0800 \mathrm{~h} .{ }^{*} \mathrm{p}<0.004 \mathrm{vs}$. $0800 \mathrm{~h} \mathrm{~B})$ FGFR1 showed peak expression at $2400 \mathrm{~h}$ and trough expression at $1200 \mathrm{~h}$.

${ }^{\star \star} p<0.006$ vs. $2400 \mathrm{~h},{ }^{\star} \mathrm{p}<0.04$ vs. $2400 \mathrm{~h}$, †Unpublished data 


\section{References}

[1] Levi-Montalcini R. The nerve growth factor 35 years later. Science 1987;237:1154-62.

[2] Barde YA, Edgar D, Thoenen H. Purification of a new neurotrophic factor from mammalian brain. EMBO J 1982;1:549-53.

[3] Thoenen $H$. The changing scene of neurotrophic factors. Trends Neurosci 1991;14:165-70.

[4] Tang JB, Chen CH, Zhou YL, McKeever C, Liu PY. Regulatory effects of introduction of an exogenous FGF2 gene on other growth factor genes in a healing tendon. Wound Repair Regen 2014;22:111-8.

[5] Yanagita M, Kojima Y, Kubota M, Mori K, Yamashita M, Yamada S, et al. Cooperative effects of FGF-2 and VEGF-A in periodontal ligament cells. J Dent Res 2014;93:89-95.

[6] Evans SJ, Choudary PV, Neal CR, Li JZ, Vawter MP, Tomita H, et al. Dysregulation of the fibroblast growth factor system in major depression. Proc Natl Acad Sci U S A 2004;101:1550611.

[7] Rubenstein JL. Annual Research Review: Development of the cerebral cortex: implications for neurodevelopmental disorders. J Child Psychol Psychiatry 2011;52:339-55.

[8] Borello U, Cobos I, Long JE, McWhirter JR, Murre C, Rubenstein JL. FGF15 promotes neurogenesis and opposes FGF8 function during neocortical development. Neural Dev 2008;3:17.

[9] Amaral DG, Schumann CM, Nordahl CW. Neuroanatomy of autism. Trends Neurosci 2008;31:137-45.

[10] Shimamura K, Rubenstein JL. Inductive interactions direct early regionalization of the mouse forebrain. Development 1997;124:2709-18.

[11] Mott NN, Chung WC, Tsai PS, Pak TR. Differential fibroblast growth factor 8 (FGF8)mediated autoregulation of its cognate receptors, Fgfr1 and Fgfr3, in neuronal cell lines. PLoS One 2010;5:e10143.

[12] Cholfin JA, Rubenstein JL. Patterning of frontal cortex subdivisions by Fgf17. Proc Natl Acad Sci U S A 2007;104:7652-7.

[13] Cholfin JA, Rubenstein JL. Frontal cortex subdivision patterning is coordinately regulated by Fgf8, Fgf17, and Emx2. J Comp Neurol 2008;509:144-55.

[14] Terauchi A, Johnson-Venkatesh EM, Toth AB, Javed D, Sutton MA, Umemori H. Distinct FGFs promote differentiation of excitatory and inhibitory synapses. Nature 2010;465:783-7. [15] Kanda T, Iwasaki T, Nakamura S, Kurokawa T, Ikeda K, Mizusawa H. Self-secretion of fibroblast growth factor-9 supports basal forebrain cholinergic neurons in an autocrine/paracrine manner. Brain Res 2000;876:22-30.

[16] Vaccarino FM, Schwartz ML, Raballo R, Nilsen J, Rhee J, Zhou M, et al. Changes in cerebral cortex size are governed by fibroblast growth factor during embryogenesis. Nat Neurosci 1999;2:848.

[17] Nandy SB, Mohanty S, Singh M, Behari M, Airan B. Fibroblast Growth Factor-2 alone as an efficient inducer for differentiation of human bone marrow mesenchymal stem cells into dopaminergic neurons. J Biomed Sci 2014;21:83.

[18] Okada-Ban M, Thiery JP, Jouanneau J. Fibroblast growth factor-2. Int J Biochem Cell Biol 2000;32:263-7.

[19] Cheng Y, Rodriguiz RM, Murthy SR, Senatorov V, Thouennon E, Cawley NX, et al. Neurotrophic factor-alpha1 prevents stress-induced depression through enhancement of neurogenesis and is activated by rosiglitazone. Mol Psychiatry 2015;20:744-54.

[20] Xue G, Yan HL, Zhang Y, Hao LQ, Zhu XT, Mei Q, et al. c-Myc-mediated repression of miR-15-16 in hypoxia is induced by increased HIF-2alpha and promotes tumor angiogenesis and metastasis by upregulating FGF2. Oncogene 2015;34:1393-406. 
[21] Numakawa T, Nakajima S, Yamamoto N, Ooshima Y, Odaka H, Hashido K, et al. Basic fibroblast growth factor induces miR-134 upregulation in astrocyte for cell maturation. Biochem Biophys Res Commun 2015;456:465-70.

[22] Forthmann B, Grothe C, Claus P. A nuclear odyssey: fibroblast growth factor-2 (FGF-2) as a regulator of nuclear homeostasis in the nervous system. Cell Mol Life Sci 2015.

[23] Zacherl S, La Venuta G, Muller HM, Wegehingel S, Dimou E, Sehr P, et al. A direct role for ATP1A1 in unconventional secretion of fibroblast growth factor 2 . J Biol Chem 2015;290:365465.

[24] Leng Y, Wang Z, Tsai LK, Leeds P, Fessler EB, Wang J, et al. FGF-21, a novel metabolic regulator, has a robust neuroprotective role and is markedly elevated in neurons by mood stabilizers. Mol Psychiatry 2015;20:215-23.

[25] Fakhri H, Ricken R, Adli M, Fajol A, Walter M, Foller M, et al. Impact of lithium treatment on FGF-23 serum concentrations in depressive patients. J Clin Psychopharmacol 2014;34:745-7.

[26] Antonellis PJ, Kharitonenkov A, Adams AC. Physiology and Endocrinology Symposium: FGF21: Insights into mechanism of action from preclinical studies. J Anim Sci 2014;92:407-13. [27] Liu P, Chen L, Bai X, Karaplis A, Miao D, Gu N. Impairment of spatial learning and memory in transgenic mice overexpressing human fibroblast growth factor-23. Brain Res 2011;1412:917.

[28] Asai T, Wanaka A, Kato H, Masana Y, Seo M, Tohyama M. Differential expression of two members of FGF receptor gene family, FGFR-1 and FGFR-2 mRNA, in the adult rat central nervous system. Brain Res Mol Brain Res 1993;17:174-8.

[29] Estival A, Monzat V, Miquel K, Gaubert F, Hollande E, Korc M, et al. Differential regulation of fibroblast growth factor (FGF) receptor-1 mRNA and protein by two molecular forms of basic FGF. Modulation of FGFR-1 mRNA stability. J Biol Chem 1996;271:5663-70.

[30] Peng H, Myers J, Fang X, Stachowiak EK, Maher PA, Martins GG, et al. Integrative nuclear FGFR1 signaling (INFS) pathway mediates activation of the tyrosine hydroxylase gene by angiotensin II, depolarization and protein kinase C. J Neurochem 2002;81:506-24.

[31] Fang X, Stachowiak EK, Dunham-Ems SM, Klejbor I, Stachowiak MK. Control of CREBbinding protein signaling by nuclear fibroblast growth factor receptor-1: a novel mechanism of gene regulation. J Biol Chem 2005;280:28451-62.

[32] Ohkubo Y, Uchida AO, Shin D, Partanen J, Vaccarino FM. Fibroblast growth factor receptor 1 is required for the proliferation of hippocampal progenitor cells and for hippocampal growth in mouse. J Neurosci 2004;24:6057-69.

[33] Zhao M, Li D, Shimazu K, Zhou YX, Lu B, Deng CX. Fibroblast growth factor receptor-1 is required for long-term potentiation, memory consolidation, and neurogenesis. Biol Psychiatry 2007;62:381-90.

[34] Thomson RE, Kind PC, Graham NA, Etherson ML, Kennedy J, Fernandes AC, et al. Fgf receptor 3 activation promotes selective growth and expansion of occipitotemporal cortex. Neural Dev 2009;4:4.

[35] Kang W, Wong LC, Shi SH, Hebert JM. The transition from radial glial to intermediate progenitor cell is inhibited by FGF signaling during corticogenesis. J Neurosci 2009;29:1457180.

[36] Richtsmeier JT, Flaherty K. Hand in glove: brain and skull in development and dysmorphogenesis. Acta Neuropathol 2013;125:469-89.

[37] Ornitz DM. FGFs, heparan sulfate and FGFRs: complex interactions essential for development. Bioessays 2000;22:108-12.

[38] Ornitz DM, Itoh N. Fibroblast growth factors. Genome Biol 2001;2:REVIEWS3005.

[39] Reuss B, von Bohlen und Halbach O. Fibroblast growth factors and their receptors in the central nervous system. Cell Tissue Res 2003;313:139-57.

[40] Kan M, Wang F, Xu J, Crabb JW, Hou J, McKeehan WL. An essential heparin-binding domain in the fibroblast growth factor receptor kinase. Science 1993;259:1918-21. 
[41] Gong SG. Isoforms of receptors of fibroblast growth factors. J Cell Physiol 2014;229:188795.

[42] Lee M, Kang Y, Suk K, Schwab C, Yu S, McGeer PL. Acidic fibroblast growth factor (FGF) potentiates glial-mediated neurotoxicity by activating FGFR2 IIlb protein. J Biol Chem $2011 ; 286: 41230-45$.

[43] Paradiso B, Zucchini S, Simonato M. Implication of fibroblast growth factors in epileptogenesis-associated circuit rearrangements. Front Cell Neurosci 2013;7:152.

[44] Gomez-Pinilla F, van der Wal EA, Cotman CW. Possible coordinated gene expressions for FGF receptor, FGF-5, and FGF-2 following seizures. Exp Neurol 1995;133:164-74.

[45] Cuevas P, Gimenez-Gallego G. Antiepileptic effects of acidic fibroblast growth factor examined in kainic acid-mediated seizures in the rat. Neurosci Lett 1996;203:66-8.

[46] Riva MA, Gale K, Mocchetti I. Basic fibroblast growth factor mRNA increases in specific brain regions following convulsive seizures. Brain Res Mol Brain Res 1992;15:311-8.

[47] Harirchian MH, Tekieh AH, Modabbernia A, Aghamollaii V, Tafakhori A, Ghaffarpour M, et al. Serum and CSF PDGF-AA and FGF-2 in relapsing-remitting multiple sclerosis: a casecontrol study. Eur J Neurol 2012;19:241-7.

[48] Sarchielli P, Di Filippo M, Ercolani MV, Chiasserini D, Mattioni A, Bonucci M, et al. Fibroblast growth factor-2 levels are elevated in the cerebrospinal fluid of multiple sclerosis patients. Neurosci Lett 2008;435:223-8.

[49] Clemente D, Ortega MC, Arenzana FJ, de Castro F. FGF-2 and Anosmin-1 are selectively expressed in different types of multiple sclerosis lesions. J Neurosci 2011;31:14899-909.

[50] Furusho M, Dupree JL, Nave KA, Bansal R. Fibroblast growth factor receptor signaling in oligodendrocytes regulates myelin sheath thickness. J Neurosci 2012;32:6631-41.

[51] Ruffini F, Furlan R, Poliani PL, Brambilla E, Marconi PC, Bergami A, et al. Fibroblast growth factor-II gene therapy reverts the clinical course and the pathological signs of chronic experimental autoimmune encephalomyelitis in C57BL/6 mice. Gene Ther 2001;8:1207-13. [52] Zhou L, Lu G, Shen L, Wang L, Wang M. Serum levels of three angiogenic factors in systemic lupus erythematosus and their clinical significance. Biomed Res Int 2014;2014:627126.

[53] Ichinose K, Arima K, Ushigusa T, Nishino A, Nakashima Y, Suzuki T, et al. Distinguishing the cerebrospinal fluid cytokine profile in neuropsychiatric systemic lupus erythematosus from other autoimmune neurological diseases. Clin Immunol 2015;157:114-20.

[54] Itoh N, Ohta H. Roles of FGF20 in dopaminergic neurons and Parkinson's disease. Front Mol Neurosci 2013;6:15.

[55] Takagi Y, Takahashi J, Saiki H, Morizane A, Hayashi T, Kishi Y, et al. Dopaminergic neurons generated from monkey embryonic stem cells function in a Parkinson primate model. $J$ Clin Invest 2005;115:102-9.

[56] Sleeman IJ, Boshoff EL, Duty S. Fibroblast growth factor-20 protects against dopamine neuron loss in vitro and provides functional protection in the 6-hydroxydopamine-lesioned rat model of Parkinson's disease. Neuropharmacology 2012;63:1268-77.

[57] van der Walt JM, Noureddine MA, Kittappa R, Hauser MA, Scott WK, McKay R, et al. Fibroblast growth factor 20 polymorphisms and haplotypes strongly influence risk of Parkinson disease. Am J Hum Genet 2004;74:1121-7.

[58] Mizuta I, Tsunoda T, Satake W, Nakabayashi Y, Watanabe M, Takeda A, et al. Calbindin 1, fibroblast growth factor 20, and alpha-synuclein in sporadic Parkinson's disease. Hum Genet 2008;124:89-94.

[59] Xu X, Wang N, Xu H, Xie A, Jiang H, Xie J. Fibroblast growth factor 20 polymorphism in sporadic Parkinson's disease in Northern Han Chinese. J Clin Neurosci 2013;20:1588-90.

[60] Otto D, Unsicker K. Basic FGF reverses chemical and morphological deficits in the nigrostriatal system of MPTP-treated mice. J Neurosci 1990;10:1912-21. 
[61] Noda M, Takii K, Parajuli B, Kawanokuchi J, Sonobe Y, Takeuchi H, et al. FGF-2 released from degenerating neurons exerts microglial-induced neuroprotection via FGFR3-ERK signaling pathway. J Neuroinflammation 2014;11:76.

[62] Timmer M, Cesnulevicius K, Winkler C, Kolb J, Lipokatic-Takacs E, Jungnickel J, et al. Fibroblast growth factor (FGF)-2 and FGF receptor 3 are required for the development of the substantia nigra, and FGF-2 plays a crucial role for the rescue of dopaminergic neurons after 6hydroxydopamine lesion. J Neurosci 2007;27:459-71.

[63] Klejbor I, Myers JM, Hausknecht K, Corso TD, Gambino AS, Morys J, et al. Fibroblast growth factor receptor signaling affects development and function of dopamine neurons inhibition results in a schizophrenia-like syndrome in transgenic mice. J Neurochem 2006;97:1243-58.

[64] Kiyota T, Ingraham KL, Jacobsen MT, Xiong H, Ikezu T. FGF2 gene transfer restores hippocampal functions in mouse models of Alzheimer's disease and has therapeutic implications for neurocognitive disorders. Proc Natl Acad Sci U S A 2011;108:E1339-48. [65] Woodbury ME, Ikezu T. Fibroblast growth factor-2 signaling in neurogenesis and neurodegeneration. J Neuroimmune Pharmacol 2014:9:92-101.

[66] Katsouri L, Ashraf A, Birch AM, Lee KK, Mirzaei N, Sastre M. Systemic administration of fibroblast growth factor-2 (FGF2) reduces BACE1 expression and amyloid pathology in APP23 mice. Neurobiol Aging 2015;36:821-31.

[67] Turner CA, Watson SJ, Akil H. The fibroblast growth factor family: neuromodulation of affective behavior. Neuron 2012;76:160-74.

[68] Takebayashi M, Hashimoto R, Hisaoka K, Tsuchioka M, Kunugi H. Plasma levels of vascular endothelial growth factor and fibroblast growth factor 2 in patients with major depressive disorders. J Neural Transm 2010;117:1119-22.

[69] He S, Zhang T, Hong B, Peng D, Su H, Lin Z, et al. Decreased serum fibroblast growth factor - 2 levels in pre- and post-treatment patients with major depressive disorder. Neurosci Lett 2014;579:168-72.

[70] Li JZ, Bunney BG, Meng F, Hagenauer MH, Walsh DM, Vawter MP, et al. Circadian patterns of gene expression in the human brain and disruption in major depressive disorder. Proc Natl Acad Sci U S A 2013;110:9950-5.

[71] Turner CA, Gula EL, Taylor LP, Watson SJ, Akil H. Antidepressant-like effects of intracerebroventricular FGF2 in rats. Brain Res 2008;1224:63-8.

[72] Jarosik J, Legutko B, Werner S, Unsicker K, von Bohlen Und Halbach O. Roles of exogenous and endogenous FGF-2 in animal models of depression. Restor Neurol Neurosci 2011;29:153-65.

[73] Elsayed M, Banasr M, Duric V, Fournier NM, Licznerski P, Duman RS. Antidepressant Effects of Fibroblast Growth Factor-2 in Behavioral and Cellular Models of Depression. Biol Psychiatry 2012.

[74] Ji XW, Wu CL, Wang XC, Liu J, Bi JZ, Wang DY. Monoamine neurotransmitters and fibroblast growth factor-2 in the brains of rats with post-stroke depression. Exp Ther Med 2014;8:159-64.

[75] Perez JA, Clinton SM, Turner CA, Watson SJ, Akil H. A new role for FGF2 as an endogenous inhibitor of anxiety. J Neurosci 2009;29:6379-87.

[76] Turner CA, Clinton SM, Thompson RC, Watson SJ, Jr., Akil H. Fibroblast growth factor-2 (FGF2) augmentation early in life alters hippocampal development and rescues the anxiety phenotype in vulnerable animals. Proc Natl Acad Sci U S A 2011;108:8021-5.

[77] Xin F, Susiarjo M, Bartolomei MS. Multigenerational and transgenerational effects of endocrine disrupting chemicals: A role for altered epigenetic regulation? Semin Cell Dev Biol 2015. 
[78] Chaudhury S, Aurbach EL, Sharma V, Blandino P, Jr., Turner CA, Watson SJ, et al. FGF2 is a target and a trigger of epigenetic mechanisms associated with differences in emotionality: partnership with H3K9me3. Proc Natl Acad Sci U S A 2014;111:11834-9.

[79] Pan RL, Xiang LX, Wang P, Liu XY, Nie L, Huang W, et al. Low-molecular-weight fibroblast growth factor 2 attenuates hepatic fibrosis by epigenetic down-regulation of Delta-like1.

Hepatology 2014.

[80] Mallei A, Shi B, Mocchetti I. Antidepressant treatments induce the expression of basic fibroblast growth factor in cortical and hippocampal neurons. Mol Pharmacol 2002;61:1017-24.

[81] Kajitani N, Hisaoka-Nakashima K, Morioka N, Okada-Tsuchioka M, Kaneko M, Kasai M, et

al. Antidepressant acts on astrocytes leading to an increase in the expression of neurotrophic/growth factors: differential regulation of FGF-2 by noradrenaline. PLoS One 2012;7:e51197.

[82] Wang T, Zeng Z, Hu Z, Zheng L, Li T, Li Y, et al. FGFR2 is associated with bipolar disorder: a large-scale case-control study of three psychiatric disorders in the Chinese Han population. World J Biol Psychiatry 2012;13:599-604.

[83] Liu X, Zhang T, He S, Hong B, Chen Z, Peng D, et al. Elevated serum levels of FGF-2, NGF and IGF-1 in patients with manic episode of bipolar disorder. Psychiatry Res 2014;218:5460.

[84] Kao CY, Hsu YC, Liu JW, Lee DC, Chung YF, Chiu IM. The mood stabilizer valproate activates human FGF1 gene promoter through inhibiting HDAC and GSK-3 activities. J Neurochem 2013;126:4-18.

[85] Terwisscha van Scheltinga AF, Bakker SC, Kahn RS, Kas MJ. Fibroblast growth factors in neurodevelopment and psychopathology. Neuroscientist 2013;19:479-94.

[86] O'Donovan MC, Norton N, Williams H, Peirce T, Moskvina V, Nikolov I, et al. Analysis of 10 independent samples provides evidence for association between schizophrenia and a SNP flanking fibroblast growth factor receptor 2. Mol Psychiatry 2009;14:30-6.

[87] Shi Y, Li Z, Xu Q, Wang T, Li T, Shen J, et al. Common variants on 8p12 and 1q24.2 confer risk of schizophrenia. Nat Genet 2011;43:1224-7.

[88] Gaughran F, Payne J, Sedgwick PM, Cotter D, Berry M. Hippocampal FGF-2 and FGFR1 mRNA expression in major depression, schizophrenia and bipolar disorder. Brain Res Bull 2006;70:221-7.

[89] Katsel P, Davis KL, Gorman JM, Haroutunian V. Variations in differential gene expression patterns across multiple brain regions in schizophrenia. Schizophr Res 2005;77:241-52. [90] Hashimoto K, Shimizu E, Komatsu N, Nakazato M, Okamura N, Watanabe H, et al. Increased levels of serum basic fibroblast growth factor in schizophrenia. Psychiatry Res 2003;120:211-8.

[91] Flajolet M, Wang Z, Futter M, Shen W, Nuangchamnong N, Bendor J, et al. FGF acts as a co-transmitter through adenosine $\mathrm{A}(2 \mathrm{~A})$ receptor to regulate synaptic plasticity. Nat Neurosci 2008;11:1402-9.

[92] Herbert C, Lassalle G, Alcouffe C, Bono F. Approaches targeting the FGF-FGFR system: a review of the recent patent literature and associated advanced therapeutic agents. Pharm Pat Anal 2014;3:585-612.

[93] Doherty P, Walsh FS. CAM-FGF receptor interactions: a model for axonal growth. Mol Cell Neurosci 1996;8:99-111.

[94] Polanska UM, Fernig DG, Kinnunen T. Extracellular interactome of the FGF receptor-ligand system: complexities and the relative simplicity of the worm. Dev Dyn 2009;238:277-93.

[95] Enevoldsen MN, Kochoyan A, Jurgenson M, Jaako K, Dmytriyeva O, Walmod PS, et al. Neuroprotective and memory enhancing properties of a dual agonist of the FGF receptor and NCAM. Neurobiol Dis 2012;48:533-45. 
[96] Pankratova S, Bjornsdottir H, Christensen C, Zhang L, Li S, Dmytriyeva O, et al. Immunomodulator CD200 Promotes Neurotrophic Activity by Interacting with and Activating the Fibroblast Growth Factor Receptor. Mol Neurobiol 2014.

[97] Bojesen KB, Clausen O, Rohde K, Christensen C, Zhang L, Li S, et al. Nectin-1 binds and signals through the fibroblast growth factor receptor. J Biol Chem 2012;287:37420-33. 
Table 1. Effect of an acute microinjection in the dentate gyrus on growth factor expression 24 hours and 1-week latert.

\begin{tabular}{lllll} 
Growth Factor & $\mathbf{2 4} \mathbf{h}$ & $\mathbf{p}$-value & $\mathbf{1 - w e e k}$ & p-value \\
\hline FGF2 & $159 \%$ increase & $\mathrm{p}<0.0001$ & n.s. & \\
FGF9 & $54 \%$ increase & $\mathrm{p}<0.0001$ & n.s. & \\
FGFR1 & $30 \%$ increase & $\mathrm{p}<0.0001$ & n.s. & \\
BDNF & $110 \%$ increase & $\mathrm{p}<0.0001$ & $41 \%$ increase & $\mathrm{p}<0.001$ \\
trkB & n.s. & & n.s. & \\
\hline
\end{tabular}

†Unpublished data 
